\title{
LOS EFECTOS DE LA AUTARQUÍA EN LA AGRICULTURA MURCIANA
}

\author{
M.. INMACULADA LOPEZ ORTIZ \\ Universidad de Alicante
}

\begin{abstract}
RESUMEN
En este articulo se trata de analizar las repercusiones de la política autárquica e intervencionista de la postguerra en la agricultura murciana. Los efectos fueron muy desiguales en función del tipo de cultivo existente en cada zona: la agricultura tradicional inte. rrumpió su proceso de regresión, mientras que la moderna frenó su ritmo de expansión. Los años de la autarquía supusieron un paréntesis en el proceso de especialización y mo. dernización que se había iniciado en la centuria anterior. Y ello se dio en el contexto de una crisis generalizada del sector, que se materializó en una reducción de la superficie de cultivo, un descenso de la producción y una caida de los rendimientos y el consumo.
\end{abstract}

\section{ABSTRACT}

In this paper I analyze the effect of the state-intervention and autarchic economic policy on agriculture in Murcia. I find that the effects were very different depending on the kind of crop existing in a zone: while traditional agriculture regression process was interrupted, modern agriculture expansion rythm became lower. I conclude that the autharchy period cam be considered as an interruption of the specialization and modernization process that had started the previous century, happening in a context of general agriculture slump, with crop surface, consum and returns decreasing at the same time.

\section{INTRODUCCIÓN}

La trascendencia que en la economía española y en el sector agricola en particular tuvo la politica autárquica e intervencionista desarrollada por el régimen surgido de la guerra civil ha sido puesta de manifiesto por diferentes au-

Revista de Histona Econoimio

Año XIV Otono-Interno $1996, N^{1} 3$ 
tores, entre ellos C. Barciela y J. M. Naredo '. Sin embargo, en todos los traba. jos que se centran en el analisis de la agricultura durante la postguerra se ha acentuado la importancia de los efectos de dicha política sobre la economia agricola de secano 2 . Por el contrario, son mucho mas desconocidas las repercusiones sobre la agricultura más moderna desde el punto de vista tecnológico, que a su vez era la que más incardinada se encontraba en el mercado exterior. Precisamente porque se trataba de una economía en la que el sector exterior jugaba un papel fundamental, el aislamiento del pais asestó un golpe terrible a sus sectores más dinámicos: la agricultura especializada de regadio y la industria agroalimentaria ligada a ella.

En este sentido, el comportamiento de la agricultura murciana durante los años cuarenta y cincuenta constituye un excelente indicador, toda vez que en esta región estan presentes dos tipos de agricultura muy diferenciados: una, la de secano, de carácter extensivo y muy vinculada a los mercados locales y regional; otra, la de regadio, intensiva y volcada al mercado exterior ${ }^{3}$. Obviamente, no todas las agriculturas de la región se vieron afectadas en la misma medida ni de la misma manera por la politica autárquica e intervencionista de los años cuarenta; por el contrario, los efectos de ésta fueron muy desiguales en función del tipo de cultivo existente en cada zona. De un lado, se sitúan cereales, leguminosas, olivos y determinadas plantas industriales como las textiles; la otra cara de la moneda son los frutales, las hortalizas, el pimiento para pimentón y el viñedo. Las repercusiones de la política autárquica fueron muy diferentes en ambas: mientras que la primera interrumpió su proceso de regresión, la segunda frenó su ritmo de expansión. Y todo ello se dio en el contexto de una crisis generalizada del sector, la cual se evidencia en todas las estadisticas disponibles y que se materializa en una reducción de la superficie de cultivo, un descenso de la producción y una caida estrepitosa de los rendimientos $y$ del consumo.

\footnotetext{
1 Pueden consultarse C. Barciela (1981)(1985) (1986) y J. M. Naredo (1981).

2 Son relevantes, en este sentido, las aportaciones sobre el sector cerealicola de C. Barciela (1981) y (1986) y J. M. Naredo (1981); y sobre el olivar, de C. Tio (1982) y de C. Gutierrez del Castillo (1983).

${ }^{3}$ El proceso de modernización de la agricultura murciana durante la segunda mitad del siglo XIX y el primer tercio del $x x$ ha sido estudiado por J. M. Martinez Carrion (1988), (1990a) y (1990b).
} 


\section{LA EVOLUCIÓN DEL SECTOR AGRÍCOLA DURANTE LA POSTGUERRA}

Las principales claves explicativas de la evolución del sector agricola durante la postguerra son: 1) La politica intervencionista adoptada por el nuevo Estado con el objetivo de alcanzar la tan «deseada» autarquia; 2) La pérdida de los mercados exteriores como consecuencia del bloqueo internacional a que fue sometido el régimen; 3) La escasez de alimentos básicos en el mercado interno y su consecuencia: el estraperlo; y 4) La falta de medios de labor y su carestia; y, como contrapartida, la abundante y dócil mano de obra disponible. Consecuencia de todo ello fue la crisis en la que se sumió el sector agricola murciano durante los años cuarenta y primera mitad de los cincuenta. Para explicar esta crisis de casi dos décadas no cabe recurrir a las tesis oficiales - «destrozos de los rojos», «climatologia adversa», «intereses mezquinos», ...-, sino que debe culparse de tal crisis a la politica agraria emprendida por el régimen franquista al terminar la guerra. En efecto, el impacto de la guerra en la región de Murcia no puede considerarse, en modo alguno, desastroso, como mantuvo la propaganda oficial, ya que esta región permaneció en la retaguardia durante todo el conflicto. La información disponible - Memoria del Servicio de Recuperación Agricola - permite sostener que la destrucción de cultivos, de medios de labor, de ganado y de instalaciones fue de escasa importancia. Por su parte, la coyuntura climática - "pertinaz sequia»- puede explicar algunas malas cosechas, pero no el descenso productivo de más de una década.

Desde el punto de vista de la estructura agraria, lo primero que hicieron las autoridades franquistas fue proceder a la devolución a sus antiguos propietarios de las fincas que habian sido ocupadas en virtud de la legislación promulgada por la II República, las cuales suponían, según los datos del Boletín del Instituto de Reforma Agraria, un total de 260.060 hectáreas en la provincia de Murcia. En este sentido, la primera medida adoptada por el Ayuntamiento de Murcia tras la «liberación» fue la de iniciar el proceso de devolución de fincas expropiadas u ocupadas durante el periodo republicano, nombrándose para tal fin una Junta Depositaria que estaba formada por los mayores propietarios de la zona y por sus representantes. El presidente era Agustín Virgili, que era el administrador de los condes de Heredia Spinola y de los marqueses de Rafal, contándose ambos entre los mayores propietarios de la cuenca del Segura. En esta devolución se incluían los productos, ganado, maquinaria y aperos que se encontraban en las fincas en el momento de efectuarse la transferencia a sus antiguos titulares, al tiempo que dichas reposiciones traían consigo la expulsión y, en la mayoria de los casos, el enjuiciamiento de los aparceros o arren- 
datarios que habian tomado parte en las colectivizaciones agrarias. Asi pues, si en algún sector quedó rápidamente en evidencia el tipo de intereses económicos que defendia el régimen triunfante de la guerra, éste fue el agricola, con la puesta en marcha de la contrarreforma, y así lo entendieron los propietarios que acudieron al Servicio Nacional de Reforma Económica y Social de la Tierra con apremiantes demandas para que les fueran devueltas sus «fincas usurpadas o invadidas por los rojos».

Se impuso en lo sucesivo un modelo de reforma agraria eminentemente técnico, cuyo objetivo principal fue la colonización -entendida como puesta en regadio, parcelación y otras mejoras técnicas-, dejando en un plano muy secundario la redistribución. La política de colonización tuvo dos vertientes principales: por un lado, lo que se denominó como política de colonización de grandes zonas y, por otro, la llamada de interés local. Sus resultados en la provincia de Murcia fueron muy desiguales ${ }^{4}$. La de grandes zonas fue prácticamente inexistente: la actuación del Instituto Nacional de Colonización, entre 1939 y 1950, se limitó a la colonización de tres fincas, con una superficie total de 321 hectáreas, en las que se instalaron 306 colonos. Las fincas adquiridas por el INC fueron «Mayorazgo de Alquerias» (1945), «El Parador» (1945) y «El Palmar» (1947). Para su adquisición se utilizó el procedimiento de ofrecimiento voluntario por parte de sus dueños, pagandose por ellas una cifra superior a los 7 millones de pesetas, lo que era una tasación muy favorable para los propietarios, ya que las tierras, que no eran de regadio efectivo ni en su totalidad productivas, fueron compradas por el INC a un precio que estaba por encima de su valor de mercado, como se reconocia en los expedientes de compra.

Muy distintos fueron los resultados de la politica de interés local, con la que se queria favorecer y potenciar la realización de mejoras rurales y, principalmente, la transformación de secanos en regadios. Murcia fue, tras Valencia, la provincia en que mayor cuantia alcanzaron los subsidios estatales concedidos entre 1939 y 1965, que en su totalidad rebasaron los 567 millones de pesetas, cantidad que en su mayor parte se concedió por el INC en forma de auxilios para financiar, a muy bajo interés, las obras de transformación y puesta en regadio emprendidas por los propietarios murcianos. El INC contribuyó de esta manera a capitalizar la agricultura murciana mediante la subvención de obras que transformaron una gran extensión de secano -más de 47.000 hectáreas - en regadio y todo ello a un coste muy bajo para los propietarios.

+ La plasmación de la politica de colonización en la región de Murcia durante la postguerra ha sido trabajada por M.' I. López Ortiz (1986); de este trabajo están sacados los datos que se ofrecen en este articulo. 
Por otra parte, la evolución del sector agricola durante la postguerra se vio mediatizada por dos fenómenos que contribuyeron a frenar su modernización tecnológica: de un lado, el espectacular incremento de la población activa en el sector y, de otro, el fuerte descenso de los medios de labor disponibles. Al finalizar la guerra y hasta los años cincuenta, se produjo un incremento notable en el número de activos, que aumentó en más de 10 puntos su participación respecto a 1930 , lo que constituye una buena muestra del proceso de ruralización sufrido por la economia murciana en estos años. La escasez y la carestía de los alimentos básicos y el estancamiento industrial hicieron que buena parte de la población que habia emigrado de las zonas rurales — principalmente de las áreas de secano- para emplearse en otras actividades retornara al ámbito rural como forma de asegurar siquiera la subsistencia. Este aumento de población activa contribuyó a frenar el crecimiento de los salarios agricolas, cuyo nivel resultó ser muy inferior al de los precios percibidos por los agricultores 5 . Este retraso en el crecimiento de los salarios en relación con los precios agrarios compensó el efecto negativo de la caida de los rendimientos sobre los beneficios agrarios. Asi, como ha señalado Naredo (1986), la rentabilidad de los empresarios agricolas pudo alcanzar, a finales de la década de 1940 y principios de la de 1950 , unos niveles muy superiores a los de los años treinta, viéndose de esta manera reforzada la acumulación de capital de origen agrario. Ahora bien, este comportamiento de los salarios agricolas no puede explicarse sólo por el aumento de la oferta de mano de obra, sino que también debe contextualizarse en el marco del nuevo reparto del poder político que resultó de la guerra. No fue hasta la década de los años cincuenta cuando se reanudaron los trasvases de población del campo a las zonas industriales, es decir, cuando se retomó la tendencia al decrecimiento de la población activa del sector agricola. En el caso de la región de Murcia, a pesar de que es muy significativa la reducción del componente agrario, su peso siguio siendo muy superior a la media nacional, lo que se justifica por el hecho de que esta región se configura como un espacio con un grado medio de industrialización y por el carácter intensivo en mano de obra que presenta la agricultura de regadio murciana.

'Segun los indices elaborados por el INIA tomando como base el periodo 1930-35, el indice de precios percibidos por los agricultores en el mercado oficial alcanza en 1951 el valor 800 , mientras que el indice de salarios se situa en 535. Pero debe tenerse en cuenta que los precios de los productos intervenidos que se utilizan son los del mercado oficial, lo que permite suponer que, dada la amplitud del mercado negro y el elevado desnivel de los precios de este respecto a los oficiales, la relación salarios precios fue mas favorable para estos últimos de lo que indican esos indices. 


\section{CUADRO 1}

Indice de salarios agricolas reales en España (base 100 en 1936)

\begin{tabular}{|c|c|c|}
\hline 1940 & 82 & 1946 \\
\hline 1941 & 73 & 1947 \\
\hline 1942 & 72 & 1948. \\
\hline 1943 & 74 & 1949 \\
\hline 1944 & 73 & 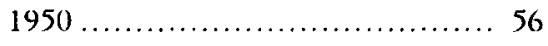 \\
\hline 1945 & 72 & \\
\hline
\end{tabular}

Futwr: Anuarios Estadisticos del INF.

\section{CUADRO 2}

Estructura de la población activa en la región de Murcia. En porcentajes

\begin{tabular}{lccc}
\hline & Sector Agricola & Sector Industrial & Sector Servicios \\
\hline $1930 \ldots \ldots \ldots \ldots \ldots \ldots \ldots$ & 49,0 & 23,3 & 27,7 \\
$1940 \ldots \ldots \ldots \ldots \ldots \ldots \ldots$ & 59,5 & 19,3 & 21,2 \\
$1950 \ldots \ldots \ldots \ldots \ldots \ldots$ & 59,3 & 18,4 & 22,3 \\
$1960 \ldots \ldots \ldots \ldots \ldots \ldots$ & 44,8 & 26,7 & 28,5 \\
\hline
\end{tabular}

FuEvl': Censor de Población.

El proceso de industrialización y urbanización de la economía española, notable ya en el primer tercio del siglo xx, habia favorecido la introducción de nuevas tecnologias ahorradoras de trabajo. Sin embargo, este proceso también se vio bruscamente interrumpido en la década de los cuarenta, debido a la imposibilidad de importar medios de producción en las cantidades necesarias. Asi, se observa un descenso en el empleo de técnicas intensivas en capital: tanto el número de tractores por activo en la agricultura como el uso de fertilizantes cayeron en relación con los años treinta. Como puede verse en el cuadro 3, durante la década autárquica se redujeron de forma significativa las cantidades de sustancias fertilizantes utilizadas en la agricultura española, en especial la correspondiente a los productos nitrogenados. Ello se debió al bloqueo de nuestras importaciones que quedaron restringidas a los envios de nitratos procedentes de Chile, que fueron durante estos años casi la única provisión de nitrógeno que se recibió desde el exterior. Por su parte, la industria nacional sólo cubria una pequeñisima parte de las necesidades de la agricultura -menos del $6 \%-$ y ello a unos precios mucho más elevados. Esta penuria hizo 
que se establecieran una serie de normas de preferencia para el uso de los abonos nitrogenados ", arbitrándose unos cupos para aquellos cultivos que constituian una parte importante de la dieta alimenticia del pais y que necesitaban estas sustancias para obtener unos rendimientos aceptables. En cuanto a los abonos fosfatados y potásicos, se consideraba que el mercado estaba suficientemente abastecido, y ello a pesar del notable descenso del consumo respecto al periodo republicano. En cualquier caso, la escasez de fertilizantes de origen mineral y quimico fue un handicap para el desarrollo de la agricul. tura, su penuria dio lugar a una revalorización del uso del estiércol y a la práctica de sembrar determinadas leguminosas que enriquecian la fertilidad de los suelos ${ }^{i}$.

\section{CUADRO 3}

\section{Evolución del consumo de fertilizantes minerales en España} (En miles de Tm)

\begin{tabular}{lcccc}
\hline & Nitrogenados & Fosfatados & Potásicos & Total Abonos \\
\hline $1931.35(*) \ldots \ldots$ & 72,9 & 163,0 & 24,9 & 260,8 \\
$1945 \ldots \ldots \ldots \ldots$ & 11,3 & 81,2 & 30,7 & 113,2 \\
$1946-50 \ldots \ldots \ldots$. & 39,0 & 98,5 & 43,4 & 180,9 \\
$1951.55 \ldots \ldots \ldots$ & 121,9 & 201,9 & 46,2 & 370,0 \\
\hline
\end{tabular}

FLenre: (") Datos calculados de las series presentadas por D. Gallego (1986) y Anuarios de Estadistica Agraria, 1974 y 1975.

También se produjo un retroceso en la modernización de los aperos agricolas. A la escasez de todo tipo de medios de labor mecánicos y de piezas de recambio se sumaron los limitados cupos de carburantes adjudicados al sector agricola para satisfacer sus necesidades y los efectos de la crisis energética que

- El orden de preferencias se estableció de la siguiente manera: al arroz se le daba absoluta prioridad en el empleo del sulfato amónico disponible, siguiendo en importancia para el uso de los restantes abonos nitrogenados cultivos como la remolacha y la caña de azucar, la patata y detcrminadas plantas horticolas como el tomate y el pimiento. Por detras se situaban las plantas textiles - lino y cañamo- y los cereales. Sólo en último lugar se mencionaban los naranjales de Levante como destinatarios de «alguna cantidad de abonos nitrogenados, dentro de la penuria actualm.

La inexistencia de datos desagrcgados por provincias referidos al consumo de fertilizantes ha hecho que me tenga que referir al consumo nacional; sin embargo, algunos datos aislados con. firman la lendencia para el caso de Murcia. 
atravesó el pais ${ }^{8}$. Esto hizo que los equipos existentes funcionaran muy por debajo de sus posibilidades. Por otra parte, la abundante y barata mano de obra cisuadió a los empresarios de invertir los capitales necesarios para mecanizar sus explotaciones, ya que resultaba más rentable acudir al excedentario mercado laboral.

En definitiva, la oferta abundante de mano de obra y la escasez de input permitieron que el sector agrario reforzara sus características de economia natural, en la que se reponian la mayor parte de la energía y las materias primas invertidas en el proceso productivo, lo que acentuó su función de exportador neto de mercancias y, con ello, su capacidad de financiación. Por el contrario, en los años cincuenta, el trasvase de activos agrarios a otros sectores y la consiguiente subida de los salarios agricolas forzó a los empresarios a sustituir mano de obra por capital, en un momento en que dicha sustitución resultaba factible dado que la oferta de input era cada vez más amplia y diversificada.

Los años de la autarquía supusieron una interrupción del proceso de especialización y modernización que se habia iniciado en la centuria anterior ${ }^{4}$, fenómeno que quedó reflejado en la estructura del uso del suelo agricola (cuadro 4), en forma de estancamiento de sus componentes durante casi dos décadas. En comparación con las cifras correspondientes a la preguerra, se aprecia una notable disminución del porcentaje destinado al sistema cereal, mientras que se detecta un apreciable incremento de los referidos a frutales, viñedo y olivar; siendo también significativos los aumentos de tubérculos y plantas forrajeras. Pero tras la guerra y hasta mediados de los años cincuenta, se produjo un cierto estancamiento de los porcentajes correspondientes a los diferentes aprovechamientos. No obstante, esta distribución porcentual del uso del suelo, útil para,detectar los cambios acontecidos en el tipo de aprovechamiento de las explotaciones, enmascara una realidad plasmada en todas las fuentes: la reducción de la superficie de cultivo en prácticamente todos los aprovechamientos, tal como veremos más adelante.

Fue en la década de los años cincuenta, al amparo de las progresivas medidas liberalizadoras, cuando se inició una política agraria diferente, que tenía como principales objetivos acabar con el mercado negro, el hambre y el subconsumo, y relanzar la actividad agricola, sentando las bases de la modernización del sector. También fue en esta década cuando se abandonaron las opera-

${ }^{8}$ En este sentido, los empresarios agrarios de la región expresaron reiteradamente las dificiles condiciones en las que se veian obligados a laborar sus explotaciones, solicitando más y mejores medios de labor.

"Sobre dicho proceso y referido a la región de Murcia pueden verse los trabajos de J. M. Martinez Carrión que aparecen citados en la bibliografia. 


\section{CUADRO 4}

Estructura del uso del suelo agricola en la provincia de Murcia

(Cifras en porcentajes)

\begin{tabular}{|c|c|c|c|c|c|c|c|c|}
\hline & $\begin{array}{l}\text { Sistema } \\
\text { cereal }\end{array}$ & Tubirculos & $\begin{array}{c}\text { Plantas } \\
\text { industriales }\end{array}$ & $\begin{array}{l}\text { Plantas } \\
\text { horticolas }\end{array}$ & Frutales & Viriedo & Olivar & $\begin{array}{l}\text { Plantas } \\
\text { gorrajeras }\end{array}$ \\
\hline 1935 (*) $^{\circ}$ & 59,8 & 0,6 & 1,1 & 1,7 & 10,9 & 15,9 & 8,8 & 1,2 \\
\hline 1940.45 & 54,8 & 1,3 & 0,6 & 1,2 & 13,1 & 16,9 & 10,3 & 1,8 \\
\hline 1946.50 & 52,9 & 1,9 & 1,2 & 0,8 & 13,4 & 17,3 & 10,6 & 1,9 \\
\hline 1951.55 & 52,4 & 1,7 & 1,9 & 1,0 & 14,0 & 16,8 & 10,3 & 1,9 \\
\hline
\end{tabular}

FufNTE: (*) Los datos base estan tomados de J. M. Martinez Carrion (1989), aunque han sido reelaborados para detraer las superficies correspondientes al barbecho en el sistema cereal y al esparto en las plantas industriales, para que fueran homologables a la serie que presento. M. l. Lopez Ortiz (1992), con datos extraidos de los Antarios Estadíti. cos de las Produciones Agrarias.

ciones asentadoras de colonos y se impulsaron las estrategias modernizadoras del agro desde el INC. Esta nueva coyuntura propició que en la región de Murcia se retomara y se profundizara en el proceso de especialización hortofruticola, lo que llevó parejo la crisis definitiva de los sectores más tradicionales. Asi, en los secanos, cereales y olivos vieron cómo retrocedía su participación en la estructura del uso del suelo a medida que crecian sus costes de oportunidad y que mejoraban las expectativas de rentabilidad de otros cultivos alternativos, como el viñedo y sobre todo el almendro. Pero fueron determinados cultivos de regadio - hortalizas, frutales de hueso y citricos - los grandes protagonistas de los cambios productivos acontecidos en el sector agricola murciano, cambios que se mostrarán irreversibles a la altura de los años sesenta.

\section{EL RELATIVO AUGE DE LOS CULTIVOS TRADICIONALES}

Como los efectos de la autarquía sobre los sectores cerealícola y oleicola son conocidos gracias a los trabajos de Barciela, Naredo, Tió y Gutiérrez del Castillo, entre otros, me referiré en este articulo tan sólo a las peculiaridades que presentan estos sectores en Murcia. Durante el primer tercio del siglo xx, cereales y leguminosas ocupaban buena parte de la superficie agricola de la region, aunque la tendencia, desde finales del siglo pasado, era claramente hacia el descenso; y algo parecido ocurria con el olivar. En efecto, el olivar murciano, que habia incrementado moderadamente su superficie de implantación en 
el primer tercio del siglo $\mathrm{xx}$, inició su regresión en los años treinta, estancándose en 30.602 hectáreas hasta mediados de los cincuenta, en que se retomó la tendencia al descenso. ¿Por qué en los años cuarenta se paralizó el proceso de sustitución de estos cultivos? Desde luego, ello no se debió a que éstos consiguieron incrementar su grado de competitividad 10: la región continuó siendo una zona poco dotada para estos aprovechamientos.

A partir de la guerra, lo más destacable no fue la reducción de la superficie ocupada por los cereales, leguminosas y olivos, sino su estabilidad hasta la década de los años sesenta (cuadros 5, 6 y 8). En este sentido, la política autárquica y la escasez de alimentos se revelaron como dos poderosos pilares que apoyaron su mantenimiento. No cabe la menor duda de que el valor de sus productos en un contexto caracterizado por la carestía y sobre todo el de aquellas partidas desviadas al estraperlo debió persuadir a los agricultores para que continuaran sembrando cereales y cultivando olivos. Asi, la participación del sistema ceréal respecto al conjunto del valor del producto agricola regional se incrementó durante las décadas de los años cuarenta y cincuenta, para, a partir de 1956, iniciar un declive definitivo. Por su parte, el elevado valor que adquirió el aceite de oliva hizo rentable el cultivo de cualquier olivar, por marginal que éste fuera. Además, aunque antes señalaba que fue la interrupción del proceso de sustitución de los cereales y de los olivos por otros cultivos el rasgo más característico de estos subsectores, hay que dejar constancia que ambas superficies de cultivo fueron significativamente menores que las existentes en el quinquenio 1931-35, y ello aun admitiendo que las estadísticas oficiales presentan ciertas limitaciones que tienen que ver con la existencia del mercado negro ${ }^{11}$. También descendió la producción y los rendimientos por unidad de superficie estuvieron por de. bajo de los niveles cosechados en los años treinta (cuadros 5, 6 y 7). Por lo que hay que destacar el profundo bache productivo de estos aprovechamientos en la década de los cuarenta.

En cuanto a la evolución seguida por los diferentes cultivos que integran el sistema cereal, hay que destacar asimismo la interrupción del proceso de

${ }^{10}$ En un estudio realizado por el profesor $M$. de Torres en los años cincuenta, este afirmaba que el cultivo del olivo en la cuenca del Segura arrojaba el producto bruto por hectárea mas bajo de todas las cuencas hidrograficas del pais donde este aprovechamiento estaba presente.

1 Como ocurrió en todo el pais, el mercado negro llevó consigo la ocultación de superficies y producciones de aquellos cultivos intervenidos; sin embargo, los descensos recogidos en las es. radisticas no pueden achacarse exclusivamente a las deficiencias estadisticas, ya que cuando se normalizo el mercado no afloraron las hectáreas ni las producciones ocultadas, sino que continúan con algunas variaciones poco relevantes las tendencias del periodo autárquico. 


\section{CUADRO 5}

Evolución de la superficie sembrada, la producción y los rendimientos del sistema cereal en Murcia

\begin{tabular}{|c|c|c|c|}
\hline & $\begin{array}{c}\text { Superticie } \\
\text { (Has) }\end{array}$ & $\begin{array}{l}\text { Producción } \\
\quad(Q m s)\end{array}$ & $\begin{array}{l}\text { Rendimientos } \\
\left(\mathrm{Q}_{\mathrm{ms} / \mathrm{Ha})}\right.\end{array}$ \\
\hline 1931.35 & 232.902 & 2.046 .740 & 8,70 \\
\hline $1939-40$ & 132.736 & 951.427 & 7,16 \\
\hline 1941.45 & 163.230 & 1.042 .157 & 6,38 \\
\hline $1946.50 \ldots$ & 153.056 & 985.110 & 6,43 \\
\hline $1951.55 \ldots \ldots \ldots \ldots$ & 156.204 & 1.160 .338 & 7,42 \\
\hline
\end{tabular}

Funte: Anuarios Estadisticos de las Producciones Agrarias.

sustitución del trigo por la cebada en los secanos de Murcia ${ }^{12}$, proceso que habia tenido como consecuencia la supremacia de la cebada sobre el trigo, dedicándose a la primera más de la mitad de la superficie cerealera. Los mayores rendimientos, su cotización a unos precios no muy inferiores a los del trigo, sus menores exigencias hídricas y el hecho de que demandaba menor número de labores fueron los motivos que indujeron a los agricultores a expandir este cultivo; sin embargo, las especiales características del periodo autárquico paralizó esta sustitución, que no se retomaria hasta que éstas desaparecieron.

\section{CUADRO 6}

Evolución de la superficie y de las producciones principales del olivar en Murcia

\begin{tabular}{lcrc}
\hline & $\begin{array}{c}\text { Supcrficic } \\
\text { (Has) }\end{array}$ & $\begin{array}{c}\text { Aceituma } \\
\text { (Qms) }\end{array}$ & $\begin{array}{c}\text { Accite } \\
\text { (Qms) }\end{array}$ \\
\hline $1931.35 \ldots \ldots \ldots \ldots \ldots$ & 34.210 & 135.512 & 27.199 \\
$1939.40 \ldots \ldots \ldots \ldots \ldots \ldots$ & 32.094 & 87.837 & 14.389 \\
$1941.45 \ldots \ldots \ldots \ldots \ldots \ldots$ & 30.602 & 87.012 & 15.272 \\
$1946-50 \ldots \ldots \ldots \ldots \ldots$ & 30.602 & 186.842 & 33.412 \\
$1951.55 \ldots \ldots \ldots \ldots \ldots$ & 30.602 & 126.945 & 22.320 \\
\hline
\end{tabular}

Ft: $\mathrm{NTt}$ : Anuarios Estadisticos de las Producciones Agrarias.

${ }^{12}$ Proceso que ya hahia sido detectado por J. M. Martinez Carrion (1990a) para las últimas décadas del siglo XIX. 


\section{CUADRO 7}

Evolución de los rendimientos del olivar y del proceso de transformación de la aceituna en Murcia

\begin{tabular}{|c|c|c|c|c|}
\hline & $\begin{array}{l}\text { Acertuna mesa } \\
\left(Q m s / H_{a}\right)\end{array}$ & $\begin{array}{c}\text { Aceituna almazara } \\
(\mathrm{Qms} / \mathrm{Ha})\end{array}$ & $\begin{array}{c}\text { Aceite } \\
\left(Q m s / / H_{a}\right)\end{array}$ & $\begin{array}{l}\text { Qms de aceite } \\
\text { Qms aceituna }\end{array}$ \\
\hline 1931.35 & 6,78 & 4,02 & 0,80 & 0,20 \\
\hline 1939.40 & 4,53 & 2,67 & 0,47 & 0,17 \\
\hline $1941.45 \ldots \ldots \ldots$ & 5,64 & 2,76 & 0,51 & 0,19 \\
\hline $1946-50 \ldots \ldots \ldots$ & 6,40 & 6,10 & 1,13 & 0,18 \\
\hline $1951.55 \ldots \ldots \ldots$ & 4.89 & 4,13 & 0,75 & 0,18 \\
\hline
\end{tabular}

Futatr: Anuarios Fsladisticos de las Producciones Agrarias

En el caso del olivar, hay que señalar que sólo una situación de escasez generalizada, como la que sufrio el pais al finalizar la guerra, hizo «rentables» los olivares de Murcia y ello no porque el mercado oficial remunerara positivamente sus productos, sino por la posibilidad de introducirlos en el mercado clandestino, aunque fuese a pequeña escala, y porque aseguraban a sus productores el consumo de aceite, que era el principal aporte de grasas a la dieta alimenticia de las zonas mediterráneas, llegando a utilizarse este producto, al igual que ocurrió con la harina y demás bienes intervenidos, como forma de pago en el comercio de trueque que se expandió por las zonas rurales.

En la región de Murcia, el cultivo de leguminosas iba asociado al de los cereales, utilizándose, además de para el consumo humano o animal, como componente nitrificante de los suelos destinados a la producción cerealera. En este sentido, el incremento de la superficie sembrada de leguminosas puede interpretarse como una doble respuesta: por un lado, encaminada a satisfacer la demanda de alimentos $y$, por otro, como estrategia para incrementar la productividad agricola en un momento de escasez de abonos, y sobre todo de nitratos. La producción se mantuvo por debajo de los niveles alcanzados en la II República, siendo el periodo en que las legumbres alcanzaron su máxima extensión también el de menor producción, lo que da buena cuenta de los bajos rendimientos obtenidos (cuadro 8). Este hecho se explica si se tiene en cuenta que la expansión de las leguminosas se localizó en tierras muy marginales, de escasa calidad productiva, y en las que se practicó un cultivo poco esmerado.

En general, los cereales y el olivar actuaron como comodin, contrayéndose o manteniéndose su cultivo en relación con la rentabilidad que las diferentes coyunturas les brindaban, en función de las expectativas gananciales de otros 


\section{CUADRO 8}

Evolución de la superficie, producción y rendimientos de las leguminosas en Murcia

\begin{tabular}{|c|c|c|c|}
\hline & Superficie (Has) & Producción (Qms) & Rendimientos $(Q m s / H a)$ \\
\hline $1931-35$ & 5.024 & 53.540 & 10,65 \\
\hline $1939-40$ & 4.827 & 30.827 & 6,38 \\
\hline 1941.45 & 9.057 & 28.257 & 3,11 \\
\hline 1946.50 & 6.965 & 32.610 & 4,68 \\
\hline $1951.55 \ldots \ldots \ldots \ldots$ & 6.632 & 33.538 & 5.05 \\
\hline
\end{tabular}

Furtate: Anuarios Frtaditicos de las Productones Agrarias

cultivos alternativos y de las necesidades de autoabastecimiento de la familia campesina. Su mantenimiento hasta los años 60 , en el contexto de una tendencia regresiva que se habia iniciado en la centuria anterior, debe relacionarse, fundamentalmente, con la excepcional coyuntura que le brindó la postguerra. Cuando esta finalizo, estos cultivos iniciaron su ocaso definitivo. Las nuevas condiciones del mercado y la escasa productividad de los secanos murcianos volvian a hacer a estos cultivos poco rentables para sus productores. Este repliegue se relaciona con el mayor coste de oportunidad de estos cultivos y con las mejores expectativas que presentaba el cultivo de frutales de secano $y$, en concreto, el almendro. La postguerra habia sido sólo un paréntesis.

\section{EL DIFERENTE COMPORTAMIENTO DE LAS PLANTAS INDUSTRIALES}

Los cultivos industriales mostraron una dinámica muy diferente según su producción se encaminara al autoconsumo nacional o a la exportación. Entre los primeros se cuentan las plantas textiles que conocieron durante la postguerra una etapa de relativo florecimiento; de los segundos, cuyo principal exponente es el pimiento para pimentón, sufrió en esos mismos años un profundo bache del que no se recuperaria hasta bien entrada la decada de los cincuenta.

Sólo la peculiar coyuntura que atravesó el pais durante los cuarenta y parte de los cincuenta explica el «resurgir» de la industria sedera y con ella del cultivo de la morera. E igual ocurrió con la industria espartera y con la expansión de los atochales. La Estación Sericícola de Murcia se convirtió en punto de mira para todos los productores del pais. No en vano, más de la mitad de las moreras cultivadas en España se ubicaban en los regadios de esta provin- 
cia, los rendimientos por arbol se contaban como los más elevados del pais, y, asimismo, los precios percibidos por los cultivadores murcianos eran los más altos ${ }^{13}$. El interes del Gobierno por este cultivo radicaba en la importancia que la producción sedera adquirio en la postguerra. Las medidas arbitradas por el régimen franquista para fomentar la sericicultura ${ }^{1+}$ contribuyeron a in. crementar de forma notable la producción de capullo de seda, como puede verse en el cuadro 9. El interés por esta fibra no residió tanto en su aplicación para la industria del vestido como en su utilización para fines militares, ya que se utilizaba en la fabricación de diferentes articulos relacionados con el equi. pamiento del ejército. Un subproducto era la hijuela, usada en cirugia y en la pesca. Toda la producción era adquirida por el Instituto de Fomento de la Producción de Fibras Textiles a un precio tasado, al que se agregaba una pri-

\section{CUADRO 9}

Evolución de la producción de seda en Murcia y en España (En kilogramos)

\begin{tabular}{lccccc}
\hline & MURCIA & ESPAÑA & & MURCIA & ESPAÑA \\
\hline $1939 \ldots \ldots$ & 125.000 & 150.000 & $1950 \ldots \ldots$ & 405.000 & 519.321 \\
$1940 \ldots \ldots$ & 259.000 & 316.410 & $1951 \ldots \ldots$ & 361.000 & 451.754 \\
$1941 \ldots \ldots$ & 331.906 & 422.575 & $1952 \ldots \ldots$ & 406.000 & 510.554 \\
$1942 \ldots \ldots$ & 398.333 & 470.116 & $1953 \ldots \ldots$ & 478.000 & 619.741 \\
$1943 \ldots \ldots$ & 339.000 & 418.451 & $1954 \ldots \ldots$ & 422.000 & 566.399 \\
$1944 \ldots \ldots$ & 458.240 & 537.125 & $1955 \ldots \ldots$ & 363.000 & 502.958 \\
$1945 \ldots \ldots$ & 418.864 & 498.981 & $1956 \ldots \ldots$ & 470.000 & 628.346 \\
$1946 \ldots \ldots$ & 298.329 & 350.509 & $1957 \ldots \ldots$ & - & 548.931 \\
$1947 \ldots \ldots$ & 340.000 & 413.951 & $1958 \ldots \ldots$ & 355.000 & 479.995 \\
$1948 \ldots \ldots$ & 312.000 & 395.853 & $1959 \ldots \ldots \ldots$ & 410.000 & 528.500 \\
$1949 \ldots \ldots$ & 368.000 & 423.392 & & & \\
\hline
\end{tabular}

Futstr: Memoras de actividades de la Cámara de Comercio, Industria y Navegación de Murcia.

${ }^{13}$ El numero de moreras de la región permaneció estable desde los años treinta hasta el ini. cio de los sesenta en unos 268.000 arboles, e igual ocurrio con su producción - cifrada en 134,000 Qms--, segun los Anuarios Estadisticos de las Producciones Agrarias.

it Se establecieron cinco zonas de fomento sericicola, ademas de la Vega del Segura -que comprendia la provincia de Murcia y parte de las de Albacere y Alicante--. considerada como zona de plena produccion. Las cinco zonas eran: 1.* Alpujarras (Almeria, Granada, Malaga y Jaéni; 2." Región Valenciana (Valencia, Castellón y norte de Alicante) y Baleares; 3.` Bajo Ebro Tarragona, Teruel, Zaragoza, Huesca, Lérida y Barcelona); 4.* Guadalquivir Medio (Sevilla, Ca. diz, Córdoba y Badajoz); y 5." Tajo, Alberche y Tiétar (Madrid, Toledo, Ávila y Caceres). En todas ellas se facilitaba a los labradores instruccion y medios de labor. 
ma con la finalidad de hacer remunerador el precio del capullo. La relevancia que alcanzaba la producción murciana en el contexto nacional queda reflejada en el citado cuadro 9; baste con decir que, por término medio, el $80 \%$ del capullo producido en España procedia de las huertas de Murcia. También se destacaba esta zona por el número y la importancia de las hilaturas de seda existentes 15.

Por su parte, la región de Murcia contaba también, en los años cuarenta y cincuenta, como una de las principales zonas esparteras del pais. Aproximadamente, la quinta parte de los atochales españoles se ubicaban en esta provincia, correspondiéndole un porcentaje de producción de alrededor del $20 \%$ del total nacional. En estas dos décadas se produjo una expansión de la superficie ocupada por esta planta (cuadro 10) y de la industria vinculada a ella. Ello fue consecuencia del aumento de la demanda interna de esta fibra, que hizo de la manufactura del esparto una actividad económica muy importante para la region, siendo el principal centro fabril del país el municipio de Cieza, situado en esta provincia. Ahora bien, si el aislamiento internacional y la autarquia auspiciaron un relativo florecimiento del negocio sedero, paralizando el proceso de descuaje de las moreras, la liberalización de los mercados y la concurrencia en ellos de todo tipo de fibras significaron la desaparición definitiva del culti-

\section{CUADRO 10}

Evolución del aprovechamiento de los espartizales murcianos

\begin{tabular}{|c|c|c|c|}
\hline & $\begin{array}{l}\text { Superficie } \\
\text { (Has) }\end{array}$ & $\begin{array}{l}\text { Productión } \\
\quad(Q m s)\end{array}$ & $\begin{array}{l}\text { Valor de la producción } \\
\text { (Miles de plas) }\end{array}$ \\
\hline 1931.35 & 107.120 & 212.520 & 1.802 \\
\hline 1939.40 & 100.000 & 180.000 & 4.725 \\
\hline $1941-45$ & 139.623 & 237.359 & 9.494 \\
\hline 1946.50 & 139.623 & 270.868 & 30.898 \\
\hline 1951.55 & 139.693 & 229.127 & 33.695 \\
\hline $1956-58$ & 139.613 & 148.920 & 39.650 \\
\hline
\end{tabular}

Flente: Anuarios Estadisticos de las Producciones Agricolas.

is Asi, dos de las cuatro hilaturas existentes en el pais se ubicaban en esta zona, siendo una de ellas la más importante de la nacion - Fábrica Grande de Sedas Orihucla 1936 cabos) - La otra se llamaba Fábrica Nucva $(736$ cabos). Las dos hilaturas restantes cran Lombart. S. A., de Gandia y Almoines ( 880 cabos), situada en Valencia, y Sedas Orihuela 1384 cabosi, en Alicante. 
vo de la morera en esta región 16 y el comienzo de un nuevo periodo de crisis para la economia espartera ${ }^{17}$, que había tenido en los años cuarenta una etapa de auténtico esplendor.

Por su parte, el algodón se convirtió en un cultivo de «interés nacional», altamente protegido por el Estado. Las medidas arbitradas por la Administración para fomentar su producción iban desde la distribución gratuita de semillas hasta la concesión de subvenciones, pasando por el asesoramiento técnico y la fijación de unos precios minimos de adquisición muy remuneradores para el agricultor. En este contexto, no resulta extraño que el mayor ritmo de crecimiento de la superficie regional dedicada a este cultivo pueda fijarse en el período comprendido entre el segundo quinquenio de los años cuarenta y la primera mitad de los sesenta, como puede verse en el cuadro 11.

\section{CUADRO 11}

Evolución de la superficie, producción y rendimientos del algadón en Murcia

\begin{tabular}{lccc}
\hline & $\begin{array}{c}\text { Superficie } \\
\text { (Has) }\end{array}$ & $\begin{array}{c}\text { Producción } \\
(\text { Qms })\end{array}$ & $\begin{array}{c}\text { Rendimientos } \\
(\text { Qms } / \mathrm{Ha})\end{array}$ \\
\hline $1945 \ldots \ldots \ldots \ldots \ldots \ldots \ldots$ & 678 & 10.170 & 15,00 \\
$1946.50 \ldots \ldots \ldots \ldots \ldots \ldots$ & 1.400 & 10.981 & 7,84 \\
$1951.55 \ldots \ldots \ldots \ldots \ldots \ldots$ & 2.806 & 28.949 & 10,31 \\
$1956-60 \ldots \ldots \ldots \ldots \ldots \ldots$ & 2.412 & 36.729 & 15,22 \\
\hline
\end{tabular}

Fuevtr: Anuarios Estadisticos de las Producciones Agrarias.

\section{LA CRISIS Y EL REAJUSTE DEL SECTOR VITIVINICOLA}

Aunque la extensión vinicola de la región de Murcia seguia siendo grande al finalizar la guerra, ésta distaba mucho de alcanzar los máximos de otros rendimientos (cuadro 13). En efecto, en los cuarenta se asiste a una reducción del

in A partir del año 1963, se inicio un proceso de progresivo descuaje de las moreras de la región, que tuvo como resultado que, en 1975, tan sólo quedaran en la región 41.295 arboles. En lo sucesivo, la morera - despojada de su función economica - tan solo tendra, un caracter ornamental.

17 A partir de 1954, decrecio notablemente la produccion de esparto, no tanto porque disminuyó la superficie de cultivo - que se mantuvo hasta los años sesenta en unas $140.000 \mathrm{Has}$ (AEPA) como por el descenso de los rendimientos, que se redujeron a la mitad, lo que da buena muestra del abandono al que se vieron relegados los atochales de la región. En los años siguientes, los espartizales se fueron abandonando a medida que la industria espartera dejaba de ser una actividad economi. camente rentable, lo que llevó parejo el desmantelamiento de las instalaciones fabriles. 
área dedicada al viñedo ${ }^{18}$; las producciones de uva para vinificación y de mosto cayeron de forma considerable en relación con el periodo republicano, como puede verse en el cuadro 12. Ahora bien, la crisis sirvió para acelerar el proceso de reajuste productivo que necesitaban los viñedos murcianos, al tiempo que consolidó la zona de especialización más desarrollada en torno a los vinos del Altiplano de Yecla y Jumilla. No en vano fue en este periodo cuando se sentaron las bases de la posterior expansión del viñedo en las tierras murcianas: se abandonaron las tierras de cultivo más tradicional, retrocedió el viñedo en cultivo asociado, y mientras la vid para vinificación se estableció en el secano, abandonando las huertas, el viñedo de mesa desapareció de los secanos localizándose en el regadio.

\section{CUADRO 12}

Evolución de la superficie y las principales producciones del viñedo murciano

\begin{tabular}{lccc}
\hline & $\begin{array}{c}\text { Superficie } \\
\text { (Has) }\end{array}$ & $\begin{array}{c}\text { Uva para vinificación } \\
\text { (Miles Qms) }\end{array}$ & $\begin{array}{c}\text { Mosto } \\
\text { (Miles Hls) }\end{array}$ \\
\hline $1931.35 \ldots \ldots \ldots \ldots \ldots \ldots$ & 60.666 & 845,0 & 553,3 \\
$1939.40 \ldots \ldots \ldots \ldots \ldots \ldots$ & 59.937 & 359,3 & 230,2 \\
$1941.45 \ldots \ldots \ldots \ldots \ldots \ldots$ & 49.937 & 402,6 & 258,2 \\
$1946-50 \ldots \ldots \ldots \ldots \ldots \ldots$ & 49.937 & 341,9 & 228,1 \\
$1951.55 \ldots \ldots \ldots \ldots \ldots$ & 49.944 & 338,2 & 231,7 \\
\hline
\end{tabular}

FUENTE: Anuarios Estadisticos de las Producciones Agrarias.

\section{CUADRO 13}

Evolución de los rendimientos del viñedo en Murcia

\begin{tabular}{|c|c|c|}
\hline & $\begin{array}{l}\text { Rendimientos agricolas } \\
\quad(Q m s \text { uva/ha })\end{array}$ & $\begin{array}{c}\text { Rendimientos industriales } \\
(\mathrm{H} / \mathrm{s} \text { mosto/ha })\end{array}$ \\
\hline 1931.35 & 13,9 & 9,1 \\
\hline 1939.40 & 6,3 & 4,0 \\
\hline $1941-45$ & 8,8 & 5,5 \\
\hline 1946.50 & 7,5 & 4,8 \\
\hline $1951.55 \ldots \ldots \ldots \ldots$ & 7,2 & 4,9 \\
\hline
\end{tabular}

Fuente: Anuarios Estadisticos de las Producciones Agrarias.

${ }^{18}$ Las estadisticas del Ministerio de Agricultura citran la extensión del viñedo de la provincia de Murcia en 59.937 hectáreas para el año 1940 , mientras que para 1941 la reducen a 49.937 . 
¿Qué fue lo que motivó la crisis del viñedo? De ninguna manera pudo ser la guerra la causa del descepamiento de 10.000 hectáreas y de la disminución de la producción viticola a menos de la mitad del volumen que se obtenia anteriormente, sobre todo si se tiene en cuenta que no fue la cosecha de 1939 la más baja. Mayores repercusiones cabe atribuir al ataque de mildiu que padecieron los viñedos españoles en 1940, epidemia que acentuó sus desastrosas consecuencias debido a la escasez de sulfato de cobre para combatirla ${ }^{19}$. No obstante, sus efectos cabe extenderlos como máximo hasta el primer quinquenio de los años cuarenta. El mildiu puede explicar una coyuntura adversa, pero no la crisis permanente del sector durante más de dos décadas. Por su parte, los factores climáticos pueden contribuir a explicar determinadas campañas negativas, pues sabemos que el viñedo es un cultivo muy sensible a las variaciones climáticas, pero igualmente éstas sólo pueden esclarecer algunos momentos concretos. Así pues, ninguno de los factores mencionados hasta ahora pueden explicar la crisis estructural del vinedo en la postguerra. Es necesario recurrir a otras causas, cuyos efectos sobre el sector fueron más duraderos y determinantes.

En primer lugar hay que referirse a la politica intervencionista que tuvo como resultado la configuración de un mercado completamente intervenido, en el que se fijaba el precio de la uva, el de los vinos y las normas para su comercialización. Este sistema, establecido por una Orden de 27 de agosto de 1940, se mantuvo hasta 1947, en que se pasó a una actitud menos intervencionista, declarándose de libre comercio la uva y el vino ${ }^{21 .}$. A pesar de que la intervención tuvo en este subsector un carácter menos relevante que la llevada a cabo en otros, motivó una respuesta por parte de los viticultores que consistió

cifra que se mantiene inalterable hasta el año 1952. Sin embargo, hay que tener en cuenta la mala calidad de las estadisticas agrarias de la época, por lo que pudo ser un error o bien pudo tratarse de un proceso gradual que se corrigió en ese año.

14 En este sentido, se afirmaba en la prensa especializada: «Las desastrosas consecuencias de los ataques de mildiu han producido alarma en todas las regiones viticolas que demandan con verdadera angustia cantidades de sulfato de cobre que superan en mucho a las existencias nacionales. Parece natural, teniendo en cuenta las dificultades de abastecimiento, tanto en cobre como de sus sales, que obtengan preferencia para su empleo aquellas regiones que por sus condiciones climatologicas estén mas expuestas a los ataques de mildium». «Aprovechamiento de medios de cultivom, en Agricultura, núm. 107 (1941), p. 81.

"La libertad de comercio se reguló por la Orden de 1 de agosto de 1946, a aplicar en la campaña 1946.47. Posteriormente, el 19 de agosto de 1947 una nueva Orden Ministerial dispuso que todos los establecimientos de venta de vinos al por menor estaban obligados a tener a disposición de los clientes un tipo de vino corriente, sano y potable, blanco o tinto, a un precio máximo fijado por el Estado. La orientación de las intervenciones del Estado no se modifico hasta la primera gran cosecha de la postguerra, que marcó la aparicion de grandes excedentes en el año 1953. 
en la no ampliación del área plantada, y esta actitud no cambió hasta que en los años cincuenta se produjo una reorientación de la política estatal ${ }^{21}$. Por su parte, las expectativas comerciales constituyen otro factor decisivo a la hora de analizar la evolución del sector vitivinicola. Desde luego, estas expectativas distaban mucho de ser halagüeñas, sobre todo en la primera década de la postguerra: el bloqueo internacional había dejado a la producción vinicola española, que ya tenia problemas de comercialización con anterioridad a la guerra, sin el mercado exterior, con excepción de los vinos de Jerez. Históricamente, la caida de la demanda externa se habia mostrado como el principal detonante de las crisis del sector ${ }^{22}$. Por otro lado, el mercado nacional tampoco presentaba incentivos para la producción vinicola. La caida de los niveles de renta de la población española hizo que se produjera una brusca reducción del consumo per cápita de vino, respecto a los niveles correspondientes al periodo republicano ${ }^{23}$.

La politica intervencionista y la caida de la demanda fueron las causas principales de la crisis de los viñedos durante la postguerra. Por ello, no es casual que fuera en la década de los cincuenta cuando se aprecia el inicio de la recuperación del sector. En efecto, cuando el Estado pasó del control de precios a garantizar la compra de los excedentes sin ningún tipo de restricción y a precios suficientemente remuneradores, se incrementó la zona de cultivo de la vid, con el consiguiente aumento de la producción. Fue, no obstante, un crecimiento de tipo extensivo, ya que hasta la década de los años setenta - una antes en el conjunto de la nación - no se vio acompañado por un alza de los rendimientos.

\section{EL PARÉNTESIS DE LA POSTGUERRA EN LA ESPECIALIZACIÓN HORTOFRUTICOLA Y EN LA MODERNIZACIÓN DE LAS HUERTAS}

La estructura de los suelos de las huertas de Murcia presentaba a comienzos del siglo $\mathrm{xx}$ notables diferencias respecto a épocas pasadas. Los cambios

21 La nueva política promovio la expansion de la superficie de los viñedos, incluso en algunas zonas de mala calidad, y ello en un contexto en el que el consumo de vino per cápita tendía a disminuir de forma importante. El problema en lo sucesivo serian los excedentes y la deprecia. ción de los productos vinicolas en un mercado regulado por la oferta y la demanda. En adelante, la intervencion estatal se orientó a evitar la expansión de la superficie plantada de vid. Así, en 1954, tras la excelente cosecha de 1953, se establecieron severas sanciones para impedir que se realizaran nuevas plantaciones de viñedo.

22 Pueden verse al respecto los trabajos de J. Pujol Andreu (1986) y T. Carnero i Arbat (1980).

2" Asi, si en el quinquenio $1931-35$ el consumo de vino en España se cifraba en 74,3 litros por habitante y año, en 1951.55 este se había reducido a 50,8 , según los datos que aporta J. R. Álvarez Rendueles (1965), p. 100 . 
introducidos en la anterior centuria se habían consolidado y afianzado en el primer tercio del $\mathrm{xx}$, ocasionando una mutación radical de los paisajes. Los cereales y demás cultivos típicos del secano desaparecieron de los regadios bien dotados, siendo sustituidos por una amplia gama de frutales y hortalizas, por tubérculos como la patata, por algunas plantas forrajeras destinadas a alimentar a la cabaña ganadera y por determinados cultivos industriales, entre los que destaca el pimiento para pimentón ${ }^{24}$. Los huertanos vieron cómo se sucedian las distintas producciones sin interrupción a lo largo de todo el año, aprovechando de esta forma las ventajas comparativas que les ofrecian las excelentes condiciones de los suelos y el clima. Sin embargo, la guerra y la postguerra significaron un paréntesis en este proceso de especialización hortofruticola que se había iniciado en la centuria anterior. La superficie ocupada por frutales y plantas horticolas se estancó - con algunas excepciones como la patata- hasta los años cincuenta, en los que se retomó, aunque con un ritmo lento, la expansión de estos cultivos (cuadro 14). Las causas fueron varias y de diversa indole, teniendo mucho que ver con la política desarrollada en estos años.

En efecto, muchas fueron las dificultades que atravesaron los sectores más dinámicos de la agricultura murciana. El retorno a una agricultura de subsistencia, la ausencia de medios de labor, la caida de la demanda de determinados productos agrarios considerados como bienes superiores y la desarticulación del comercio exterior de España al que se dirigian en buena parte estos productos, hicieron que no se ampliara su superficie de cultivo. Por el contrario, desde mediados de los cincuenta, ésta se fue expandiendo progresivamen. te a medida que la demanda de sus producciones crecia y que la adquisición de medios de labor no presentaba ningún problema. La demanda exterior de frutas y hortalizas se contrajo notablemente como consecuencia, primero, del estallido de la II Guerra Mundial y, posteriormante, del bloqueo comercial decretado por los paises occidentales al régimen antidemocrático del general Franco. Esta pérdida de los mercados externos desincentivó el cultivo de estas especies que tradicionalmente habian orientado su producción hacia el comercio internacional

Tampoco el mercado nacional ofrecia en esos años mejores expectativas. Por un lado, los años cuarenta fueron un periodo especialmente dificil para las industrias conserveras, que junto con las exportaciones en fresco eran el otro gran destino de la producción hortofruticola. Las conserveras tuvieron en estos años serios obstáculos para su desarrollo, ya que adolecian de materias

${ }^{24}$ Al respecto, pueden consultarse los trabajos de J. M. Martinez Carrión citados en la bi. bliografia. 
CUADRO 14

Evolución de la superficie ocupada por los frutales

y por los cultivos intensivos de huerta en Murcia

(En Has)

\begin{tabular}{lcccc}
\hline & Frutales & $\begin{array}{c}\text { Tubérculos, } \\
\text { raices } y \text { bulbos }\end{array}$ & $\begin{array}{c}\text { Plantas } \\
\text { borticolas }\end{array}$ & $\begin{array}{c}\text { Plantas } \\
\text { forrajeras }\end{array}$ \\
\hline $1931.35 \ldots \ldots \ldots$ & 41.558 & 2.187 & 7.290 & 4.374 \\
$1939.40 \ldots \ldots \ldots$ & 38.706 & 2.916 & 4.111 & 5.608 \\
$1941-45 \ldots \ldots \ldots$ & 38.823 & 3.346 & 3.393 & 5.240 \\
$1946.50 \ldots \ldots \ldots \ldots$ & 38.689 & 5.370 & 2.425 & 5.620 \\
$1951.55 \ldots \ldots \ldots \ldots$ & 41.709 & 5.039 & 3.076 & 5.692 \\
\hline
\end{tabular}

Funtr: Anturios Estadisticos de las Produciones Agrarias

primas tan imprescindibles como hojalata y azúcar, por no hablar de maquinaria y de repuestos para la misma, a lo que hay que sumar los graves problemas para comercializar sus productos. Por otra parte, la situación de escasez de alimentos básicos hizo que el consumo de frutas, que ya era de por si escaso, se limitara a los grupos sociales de mayor renta. La dieta espanola se caracterizó hasta mediados de los años cincuenta por ser pobre y escasamente diversificada, teniendo las frutas y sus derivados una presencia muy reducida en ella. Así, no resulta extraño que el consumo interno de frutas fuese muy modesto y en ningún caso supliera la falta de demanda externa. De otro lado, tanto los frutales de regadio como las plantas horticolas son cultivos intensivos que exigen para su normal desarrollo vegetativo un elevado consumo de inputs, por lo que son muy dependientes del mercado, ya que en él se abastecen de esos insumos básicos. La escasez de medios de labor -fertilizantes, fitosanitarios, maquinaria, combustible, etc.- fue un hecho evidente durante los años cuarenta y sus repercusiones negativas fueron más acentuadas en estos cultivos ${ }^{25}$.

Desde el punto de vista institucional, tampoco era éste un buen momento para seguir expandiendo los frutales. La política agraria estatal caminaba por otros derroteros y no sólo no auspiciaba la recuperación del sector, sino que la

${ }^{25} \mathrm{Al}$ respecto, es bastante significativa una editorial titulada «Dificultades del campom, publicada en la revista Surco (num. 4, 1942, pag. 2): «Habremos de recordar las dificultades que siguen entorpeciendo la buena marcha de nuestra producción agraria. En primer término, la insuficiencia de abonos, que siguen siendo seria preocupacion, asi como tambien las dificultades de abastecimiento de anticriptogámicos y antiparasitarios. Tampoco de ganado andamos sobrados para el laboreo, y las insuficientes cantidades de combustibles liquidos (gasolina y gas-oil) no han permitido el trabajo a fondo ni el riego abundante». 
impedia abiertamente. Ante la escasez de alimentos básicos, la política agraria se orientó hacia la expansión de las superficies dedicadas a la obtención de estos productos, llegándose a calificar de «interés bastardom la dedicación de tierras a otros cultivos que no estuviera encaminada al indicado objetivo ${ }^{26}$. En el caso de los agrios se limitó la expansión de los plantios mediante una serie de disposiciones que obligaban a los agricultores a obtener una autorización previa del Ministerio de Agricultura para establecer nuevas plantaciones o reponer las antiguas ${ }^{27}$. Por otra parte, aunque la producción de frutas y hortalizas no estuvo sometida a racionamiento, su comercialización sí que estuvo intervenida durante los años cuarenta mediante normas que limitaban la libertad de comercio ${ }^{28}$.

\section{CUADRO 15}

\section{Superficie ocupada por los principales frutales en Murcia (En Has)}

\begin{tabular}{|c|c|c|c|c|c|}
\hline & Almendro & Naranjo & Limonero & Albaricoquero & Melocotonero \\
\hline $1931-35$ & 31.043 & 4.561 & 1.088 & 1.326 & 440 \\
\hline 1939.40 & 28.214 & 4.849 & 1.549 & 1.297 & 486 \\
\hline $1941-45$ & 28.214 & 4.765 & 1.717 & 1.297 & 486 \\
\hline $1946-50$ & 28.214 & 4.765 & 1.717 & 1.297 & 486 \\
\hline $1951.55 \ldots \ldots \ldots$ & 29.135 & 5.003 & 2.284 & 1.351 & 503 \\
\hline
\end{tabular}

Fufnte: Anuarios Estadisticos de las Producciones Agrarias.

26 Así, se afirmaba en una proclama publicada en la prensa con fines propagandisticos: "La. brador: Piensa primero en España y despues en tu interes. Lo que tú siembras calma el hambre de tus hermanos los españoles. Lo que dejas de sembrar, quizá obedeciendo a un interés bastardo. perjudica a todos y no beneficia a nadie. ¡Piensa y obra, pero actua bien! El trigo es harina: la harina es pan; el pan es alimento indispensable e insustituible; la falta de pan es el hambre; el hambre es la desolación y la ruina de la Patria. ePuedes, por codicia, dejar de sembrar trigo, para beneficiarte con otros cultivos menos interesantes, aunque sean mas productivos para tí?». (Surco, num. 2,1942, p. 191.

27 La primera de estas disposiciones fue el Decreto de 14 de diciembre de 1942 , el cual se habia inspirado, según se decia en su preambulo, «en la conveniencia de extender la superficie dedicada a cereales y concentrar, en beneficio de cultivos de base para la alimentacion humana, el empleo de los escasos fertilizantes nitrogenados disponibles».

${ }_{28}$ Asi, quedó expresamente prohibida la circulación de frutas sin quias, que debian ser certificados por los presidentes de las Hermandades de Labradores. También se prohibio la libre exportación de toda clase de frutas, siendo ésta realizada exclusivamente por el Sindicato de Frutas y Productos Horticolas bajo el control directo del Gobierno Civil correspondiente, que era el organismo encargado de autorizar la exportación, por lo que los productores debian entregar su producción para la exportación al Sindicato percibiendo el importe de la misma a precio de tasa; posteriormente el Sindicato les abonaba la diferencia con el precio conseguido en el punto de destino. 
No obstante, los agricultores se comportaron en estos años siguiendo unos criterios más acordes con sus intereses personales que con la discutible lógica estatal y, desde luego, incumplieron estas disposiciones siempre que les fue posible, en especial las que restringian el uso de fertilizantes. No se arrancaron frutales por mala que fue la coyuntura para sembrar cereales. Si se estancaron los plantios - véase el cuadro 15-, fue atendiendo a la realidad socio-económica del momento que hacia que estos cultivos, intensivos en capital y trabajo, no fuesen todo lo remuneradores que podian llegar a ser en un contexto donde el mercado estuviese normalizado. Fue, por lo tanto, un comportamiento racional desde el punto de vista económico.

También fue incuestionable la caida de la producción hortofruticola tras la guerra civil, descenso que estuvo motivado por la caida de los rendimientos medios, que estuvieron muy por debajo de los cosechados en el quinquenio 1931-3529. El descenso productivo de los frutales fue espectacular - como puede verse en el cuadro 16-, siendo especialmente desfavorables los años del segundo quinquenio de los cuarenta, en los que se consiguieron la producción y los rendimientos más bajos del siglo, lo que no resulta extraño si se suman a los negativos condicionantes de la postguerra los devastadores efectos de heladas, sequia e inundaciones. A pesar de todo, las exportaciones de frutas y en especial las de los citricos continuaron siendo un pilar importante de nuestra cconomia - vease el cuadro 17- Las exportaciones de naranjas y limones contribuyeron a reducir el déficit de nuestra balanza de pagos, convirtiéndose en el más firme sostén de nuestro comercio exterior de productos agrícolas. La situación de España en los mercados internacionales, tras la gue. rra, no tenia nada que ver con el papel desempeñado en las tres primeras décadas del siglo xx. En un contexto de considerable competencia internacional, resultó decisiva la escasa participación de España en el mercado durante los años 1935 a 1939 - primero, por la helada del invierno de 1936 y, después, como consecuencia de la guerra-, ya que esta circunstancia fue aprovechada

${ }^{24} \mathrm{Al}$ respecto puede verse el siguiente cuadro, en el que se recoge la evolución de los rendimientos medios (Qms/Ha) del naranjo y limonero:

\begin{tabular}{|c|c|c|}
\hline & Naranjo & Limonero \\
\hline 1931.35. & 206,9 & 233,0 \\
\hline 1939.40 & 107,2 & 199,8 \\
\hline 1941.45 & 103,0 & 79.9 \\
\hline 1946.50 & 94,6 & 92,9 \\
\hline 1951.55. & 123.2 & 119,5 \\
\hline
\end{tabular}

Fundr: Anuarios Estadisticos de las Producciones Agrarias. 
por los países competidores para expandir su área de implantación comercial y establecer relaciones de clientela. A los problemas propios del pais se sumaron los derivados del estallido de la II Guerra Mundial, en la que participarian en mayor o menor medida todos los paises que constituian los principales centros de consumo de nuestras frutas. Asi, el periodo comprendido entre 1940 y 1948 es el que registra el volumen de exportaciones más bajo de todo el siglo. El comercio exterior se limitó durante el conflicto a los paises de Europa Central - principalmente Alemania - y al envío de algunas partidas a los paises nórdicos; aunque no se llegó a interrumpir en su totalidad el comercio de agrios con el Reino Unido y con otros paises aliados, éste si quedó reducido a pequeñas cantidades ${ }^{30}$. En los años cincuenta, el proceso de especialización hortofruticola regional se reanudó La expansión de los frutales, timida durante la primera mitad de la década, cobró fuerza en la segunda, incrementando su ritmo de crecimiento en el transcurso de las décadas siguientes. Los cambios que se produjeron en la orientación de la política económica de España y la superación por parte de los paises europeos de las dificultades económicas de la postguerra mundial tuvieron, esta vez, una repercusión positiva en el sector.

\section{CUADRO 16}

Evolución de la producción de los principales frutales en Murcia

(En miles de Qms)

\begin{tabular}{lcccc}
\hline & Almendro & Naranjo & Limonero & Albaricoquero \\
\hline $1931.35 \ldots \ldots \ldots$ & 152,5 & 943,4 & 253,5 & 263,7 \\
$1939-40 \ldots \ldots \ldots$. & 103,4 & 519,8 & 239,6 & 209,4 \\
$1941-45 \ldots \ldots \ldots$. & 126,9 & 490,8 & 137,6 & 272,4 \\
$1946-50 \ldots \ldots \ldots$ & 142,5 & 450,6 & 159,9 & 239,0 \\
$1951-55 \ldots \ldots \ldots$ & 155,9 & 616,2 & 239,2 & 244,4 \\
\hline
\end{tabular}

Fuente: Amuarios Estadisticos de las Producciones Agrarias.

Por otra parte, uno de los principales obstáculos que bloqueaba la expansión hortofruticola de la región de Murcia, cual era la escasez e irregularidad

30 En 1940 , de un total de 324.737 toneladas de naranjas exportadas, 270.000 tuvieron como destino Alemania, 40.000 Inglaterra y las 14.000 restantes se distribuyeron entre los paises nórdicos y Suiza. Las exportaciones murcianas, que ascendieron a 21.440 roneladas, se repartieron de la siguiente manera: 20.000 a Alemania, 1.400 a Inglaterra y las 40 restantes entre Francia, Noruega y Suiza. 
de los recursos hidráulicos disponibles, atenuó sus efectos con la entrada en funcionamiento de los embalses del Cenajo y del Camarillas (1960), que suponen el final de las obras de regulación de la cuenca del Segura y la posibilidad de ampliar los regadios en todas las vegas y de redotar los que contaban con caudales insuficientes. También significó el que por primera vez las huertas de Murcia dispusieran no sólo de unos recursos adecuados, sino ordenados en el tiempo, lo que las hacia aptas para producir cultivos intensivos. La politica hidráulica se mostraba decisiva para el desarrollo económico regional, en especial, para la dinámica del sector agrario más modernizado ${ }^{31}$. Simultáneamente a la ampliación de las dotaciones superficiales, se produjo una mayor explotación de las aguas subterráneas, ya que los avances técnicos de este siglo permitían acceder cada vez más a los acuíferos profundos.

\section{CUADRO 17}

Evolución de las exportaciones de naranja $(\mathrm{Tm})$

\begin{tabular}{llll}
\hline & & España & Murcia \\
\hline $1931-35 \ldots \ldots \ldots \ldots \ldots \ldots \ldots \ldots$ & 859.600 & 103.152 \\
$1940-45$ & $\ldots \ldots \ldots \ldots \ldots \ldots \ldots \ldots$ & 263.898 & 21.406 \\
$1946-50$ & $\ldots \ldots \ldots \ldots \ldots \ldots \ldots \ldots \ldots$ & 299.345 & 36.528 \\
$1951-55$ & $\ldots \ldots \ldots \ldots \ldots \ldots \ldots \ldots$ & 800.015 & 79.363 \\
$1956-60$ & $\ldots \ldots \ldots \ldots \ldots \ldots \ldots \ldots$ & 624.326 & 85.705 \\
\hline
\end{tabular}

Funte: Los datos de España están extraidos de los Anuarios Lstadísticos de las Producciones Agricolas y los de Murcia de la documentación del Sindicato Vertical de Frutas y Hortalizas.

\section{CONCLUSIONES}

Al finalizar la guerra civil, el sector agrícola de la región de Murcia se vio aquejado por graves problemas, interrumpiéndose el proceso de especialización y de modernización que se habia iniciado en la centuria anterior. La superficie labrada disminuyó, la producción y los rendimientos también cayeron y descendió el nivel de consumo per cápita de productos alimenticios. Y todo ello, a pesar de que el impacto de la guerra en esta región no habia sido, en

${ }^{31}$ Sobre la incidencia de la politica hidraulica en la economia de la cuenca del Segura, véase J. Melgarejo (1995). 
modo alguno, desastroso, como mantuvo la propaganda oficial. La causa fundamental, aunque no exclusiva, de esta crisis fue la política agraria desarrollada a lo largo de la década de los años cuarenta, fuertemente intervencionista y de signo autárquico. Por una parte, los rigidos mecanismos arbitrados para controlar producciones y precios tuvieron como resultado la aparición de un amplio mercado negro de los productos intervenidos. Los cultivos agricolas más afectados por la intervención estatal fueron los cereales, las leguminosas y el olivar, cuyas producciones constituian la base de la alimentación de la población y de la cabaña ganadera. Por otra parte, el aislamiento internacional del pais provoco un verdadero colapso de nuestro comercio exterior. Lógicamente, fueron los sectores más dinámicos de la agricultura murciana los más afectados por esta pérdida de relaciones con el exterior. No en vano, el importante papel que jugaba la demanda externa en la producción de frutas y hortalizas hacia que tanto las buenas como las malas coyunturas tuvieran su origen en el exterior; en este caso, se saldó con un fuerte retroceso de las exportaciones de los productos hortofruticolas y de sus principales derivados. Asi, los principales productos exportables - cítricos y demás frutas, bulbos y hortalizas, conservas vegetales y pimenton- fueron perdiendo mercados en beneficio de las producciones de otros paises competidores, y ello en un momento en que el mercado interior no estaba en condiciones de compensar la pérdida de los centros de comercialización extranjeros. Pero, además, el mercado exterior resultaba decisivo para conseguir los insumos necesarios para la actividad agricola, tales como maquinaria, fertilizantes, productos fitosanitarios, etc. La escasez de todo tipo de factores de producción fue una realidad a lo largo de toda la década y esta situación se agravó aún más con el control ejercido por la Administración sobre los existentes, ya que significó la aparición de un mercado negro de los mismos.

En definitiva, la caida de la demanda externa y la falta de medios de labor frenaron la expansión de la superficie hortofruticola de la región y desincentivaron su cultivo, por lo que no resulta extraño que fueran los sectores económicos ligados a esta producción los primeros en reclamar medidas que liberalizaran la economia y que fuera la oferta y la demanda la que regulase el mercado. Por el contrario, determinados cultivos de carácter tradicional - como eran los cereales, el olivo, el esparto, la morera - interrumpieron su tendencia regresiva en la ocupación del espacio regional, aunque ello no conJlevó un aumento de la producción en la mayoria de los casos. La explicación de este fenómeno reside en el valor añadido que suponía para sus cultivadores la posibilidad de desviar ciertas partidas al estraperlo o a su propio autoconsumo, en un momento de escasez y carestía de los alimentos. El estraperlo se 
convirtió para unos en un lucrativo negocio y para otros en una forma de supervivencia. Por último hay que señalar que el fuerte incremento de la población dedicada a la actividad agricola, con la consiguiente depreciación de los salarios, propició la revalorización del cultivo directo y permitió un importante proceso de acumulación de capital en el sector agrario, que sería destinado, en parte, a financiar el proceso de desarrollo industrial, sobre todo de determinadas industrias derivadas del sector, conserveras y pimentoneras fundamentalmente.

\section{BIBLIOGRAFIA}

Abad Salchoo, E., y otros (1970): Trasvase y ordenación agricola y ganadera. Hermandad Sindical de Murcia.

Alsarez Revithles, J. R. (1965): «Necesidad de una politica selectiva para la produccion vitivinicola", $I C E$, n." 387 .

BARCHLA Lolyz, C. (1981): «El estraperlo de trigo en la postguerra", Moncda y Crédito, ก." 154.

- (1985): «Intervencionismo y crecimiento agrario en España, 1936-1971", La nueva bistoria económica én España, Madrid, Tecnos.

- (1986): "Introducción», Historia agraria de la España contemporánea. 3. Ll fin de la agriculiura tradicional (1900-1960), Barcelona, Critica.

CARnfro 1 ARBAT, T. (1980): Expansión vinicola y atraso agrario, 1870-1900, Madrid, Ministerio de Agricultura.

Gallfago Maritinez, D. (1986): «Transformaciones técnicas de la agricultura española en el primer tercio del siglo $\mathrm{xx}$, Historia agraria de la España contemporánea. 3. El fin de la agricultura tradicional (1900-1960), Barcelona, Critica.

Gumerrez del. Castilio, C. (1983): «Una estimación del mercado negro de aceite de oliva en la postguerra españolà, Agriculiura y Sociedad, n." 29.

LOPLZ. ORTI\%, M. ${ }^{2}$ I. (1986): La postguerra como marco para la demagogia agrarista del franquismo: La política de colonización. Tesis de licenciatura inédita.

1992): La agricultura murciana durante el franquismo. Tesis doctoral inedita.

Martine\% Carrion, J. M. (1988): «Cambio agricola y desarrollo capitalista. El sector agrario murciano a fines del siglo XIX (1870-1914)», La crisis agraria de finales del siglo xix, Barcelona, Critica.

- (1989): «El uso del suelo y la producción agraria en la Región de Murcia, 1890-1935. Una valoración critica de las estadisticas», Cuadernos de Economia Murciana, n." 4.

(1990a): Crecimiento económico y desarrollo agrario en la Región de Murcia, 1870-1935. Universidad de Murcia (microfichas).

- (1990b): «El cambio técnico de la agricultura murciana durante el siglo XIX», Cuadernos de Economía Murciana, n." 6.

Mil.cireio Moreno, J. (1995): La intervención del Estado en la cuenca del Segura. Alicante, Juan Gil Albert. 
NarfDo, J. M. (1981): «La incidencia del "estraperlo" en las grandes fincas del sur de España", Agricultura y Sociedad, n." 19.

- (1986): «La agricultura española en el desarrollo económico», Historia agraria de la España contemporánea. 3. El fin de la agricultura tradicional (1900-1960), Barcelona, Critica.

PuIOL ANDRLU, J. (1986): "Las crisis de sobreproducción en el sector vitivinicola catalán, 1892-1935", Historia agraria de la España contemporánea. 3. El fin de la agricultura tradictonal (1900-1960), Barcelona, Critica.

Tıо, C. (1982): La política de aceites comestibles en la España del siglo xx, Madrid, Ministerio de Agricultura. 